\title{
BIODIVERSIDADE COMO SUSTENTABILIDADE: PLANTAS ALIMENTÍ́CIAS NÃO CONVENCIONAIS (PANC) NA ALIMENTAÇÃO ESCOLAR
}

\section{BIODIVERSITY AS SUSTAINABILITY: NON-CONVENTIONAL FOOD PLANTS (PANC) IN SCHOOL FEEDING}

\author{
Mariana Grisa SIMONETTI ${ }^{1}$ \\ Luciana Oliveira de FARIÑA ${ }^{2}$ \\ https://orcid.org/0000-0002-5466-4887
}

\begin{abstract}
Resumo: Trata-se de um artigo de revisão bibliográfica que aborda a importância de mercados alternativos que possibilitem a construção da sustentabilidade no sistema agroalimentar atual, partindo de políticas públicas como o Programa Nacional de Alimentação Escolar - PNAE. Seu objetivo é verificar a possibilidade de inclusão de Plantas Alimentícias Não Convencionais (PANC) na alimentação escolar. Neste contexto, o artigo discute as inter-relações com a agricultura familiar e com os circuitos locais de comercialização e a valorização da biodiversidade na alimentação. Como resultado tem que, a aproximação da agricultura familiar de programas caracterizados pelo consumo regular de alimentos, como o PNAE, pode ser uma alternativa viável para dar sustentabilidade à produção e aos produtores, valorizando as práticas agroalimentares locais e os cultivos diversificados. Recomenda-se a realização de estudos que avaliem como se dá a inclusão das PANC no PNAE no sentido da estruturação desse sistema sustentável de produção e distribuição de alimentos.
\end{abstract}

Palavras Chave: Alimentação Escolar. Agricultura Familiar. Mercados alternativos. Biodiversidade. Sustentabilidade.

\begin{abstract}
This is a literature review article that addresses the importance of alternative markets that enable the construction of sustainability in the current agri-food system, based on public policies such as the National School Feeding Program PNAE. Its objective is to verify the possibility of including Unconventional Food Plants in school meals. In this context, the article discusses the interrelationships with family farming and local marketing circuits and the valuation of biodiversity in food. As a result, bringing family farming closer to programs characterized by regular food consumption, such as the $P N A E$, may be a viable alternative to give sustainability to production and producers, valuing local agrifood practices and diversified crops. Studies are recommended to evaluate how plants is included in PNAE in order to structure this sustainable food production and distribution system.

Key Words: School Feeding. Family farming. Alternative Markets. Biodiversity. Sustainability.
\end{abstract}

\footnotetext{
${ }^{1}$ Mestranda em Desenvolvimento Rural Sustentável na Universidade Estadual do Oeste do Paraná Campus Marechal Cândido Rondon, PR. mariana_grisa@hotmail.com.

2 Prof ${ }^{a}$ Dra do Programa de Pós-Graduação em Ciências Farmacêuticas e do Programa de PósGraduação em Desenvolvimento Rural Sustentável - Campus Cascavel/ Marechal Cândido Rondon, PR. luleal32@yahoo.com.br
} 


\section{International Journal of Environmental Resilience Research and Sciente (IJERRS)}

Revista Internacional Resiliência Ambiental Pesquisa e Ciência Saciedade 5.0 Resiliência Ambiental

\section{INTRODUÇÃO}

No Brasil, grande parte do abastecimento do mercado interno é feito pela Agricultura Familiar, com uma vasta gama de produtos que compõem a dieta básica alimentar da população (BRASIL, 2006). Entretanto, no contexto das políticas públicas a agricultura familiar ainda é marginalizada, já que as políticas são quase sempre, direcionadas ao modelo agrícola dominante.

Como característica do modelo familiar de produção destaca a estreita relação entre trabalho e gestão, um modelo de produção conduzido pelos proprietários, a diversificação produtiva, o respeito dos recursos naturais e qualidade de vida, a utilização do trabalho assalariado em caráter complementar e a tomada de decisões ligadas à imprevisibilidade do processo produtivo (FAO/INCRA, 1994 apud OLALDE, 2012).

Por estar presente a multifuncionalidade na agricultura familiar, tem-se observado que, além da produção de alimentos e matérias-primas, ela também favorece o emprego de práticas produtivas mais equilibradas, como diversificação do cultivo, menor uso de insumos e preservação da natureza (OLALDE, 2012).

Desta forma, a agricultura familiar, por atender aspectos sociais e ambientais, é vista como determinante para o desenvolvimento rural sustentável, uma vez que procura equilibrar as dimensões econômica, social e ambiental do desenvolvimento. Os modos de produção da agricultura familiar no contexto da Agroecologia e Agrobiodiversidade são os conhecimentos que abrangem a sustentabilidade da agricultura.

De um ponto de vista agroecológico, a "sustentabilidade" e/ou "desenvolvimento rural sustentável", não é um conceito absoluto, mas, ao contrário, só existe mediante contextos gerados como articulação de um conjunto de elementos que permitem a perdurabilidade no tempo dos mecanismos de reprodução social e ecológica de um etnoecossistema. Entre outros a potencialização da diversidade local, tanto biológica como sociocultural (GLIESMANN, 2000). 


\section{International Journal of Environmental}

\section{Resilience Research and Science (IJERRS)}

Revista Internacional Resiliência Ambiental Pesquisa e Ciência Saciedade 5.0 Resiliência Ambiental

A partir dessa premissa, há que se pensar em ações para modelos de desenvolvimento rural que tenham o conhecimento local como elemento transformador e estratégico em direção a formas mais respeitosas de produção.

Nesse contexto, vem à tona os alimentos subutilizados, ou então, denominados de Plantas Alimentícias Não Convencionais (PANC), são espécies e variedades de plantas que foram negligenciadas pela agricultura convencional; ainda não possuem valor comercial e pertencem a um grupo grande de espécies domesticadas, semi-domesticadas ou silvestres. Incluem pequenas culturas adaptadas localmente, cultivadas em hortas caseiras, em pequenos terrenos de subsistência ou em margens de fazendas. Atualmente estão ganhando reconhecimento em função do seu potencial para aumentar a resiliência e adaptação da produção agrícola e de sistemas alimentares locais (PADULOSI, THOMPSON, RUDEBIER, 2013; KINUPP \& LORENZI, 2014).

As chamadas plantas "daninhas" (ruderais) ou "plantas do mato" (silvestres) podem ser fontes complementares de alimentos interessantes para assentamentos humanos de porte pequeno a médio e nas grandes cidades, as populações da periferia e dos arredores, também podem fazer uso destas plantas espontâneas comestíveis. A conservação da diversidade de espécies de plantas comestíveis é chave para 0 abastecimento de alimentos, especialmente para populações mais pobres e com menos terra (KINNUP, 2007).

Plantas tradicionais e adaptadas localmente podem crescer com um uso mínimo de insumos agrícolas, têm qualidades características que correspondem às necessidades locais e frequentemente desempenham papel importante nas crenças culturais, valores e atividades da população rural (GIZ, 2016; FAO 2016).

Propriedades de diferentes tamanhos e características podem se beneficiar e evoluir com a adoção de sistemas agroecológicos diversificados. Produtores de pequena escala que já utilizam práticas agroecológicas para sua própria subsistência (consumo próprio), requerem assistência para expandir suas habilidades para, de 


\section{International Journal of Environmental}

\section{Resilience Research and Science (IJERRS)}

Revista Internacional Resiliência Ambiental Pesquisa e Ciência Sociedade 5.0 Resiliência Ambiental

maneira sustentável, aumentar e diversificar sua produção e se conectar aos mercados. Na outra ponta, a agricultura industrial de larga escala requer uma transformação para melhor integrar a biodiversidade e práticas agroecológicas. Nos dois casos, é possível atingir sistemas mais diversificados, rentáveis e sustentáveis de produção de alimentos (IPES-Food, 2016). Em geral, o aproveitamento do potencial da biodiversidade pode resultar no aprimoramento simultâneo da agricultura, nutrição e modos de vida de produtores rurais.

Em relação ao mercado, a procura por esse tipo de produto vem se mostrando crescente e promissora em todo o país através dos principais canais de comercialização, como feiras, lojas especializadas, unidades de alimentação e nutrição e o mercado institucional, no qual o Programa Nacional de Alimentação Escolar (PNAE) é o principal exemplo. Cabe aqui destacar o papel da escola na formação de valores ligados aos hábitos alimentares e a nutrição para promoção da saúde a partir do contexto da sustentabilidade.

A agricultura intensificada de larga escala e a homogeneização da produção de alimentos modificaram o tipo, a qualidade e a quantidade de alimentos de muitos consumidores. Alimentos frescos produzidos localmente foram substituídos por alimentos processados mais baratos e fáceis de comer, mas geralmente mais ricos em gorduras, açúcar e/ou sal, como doces, refrigerantes, petiscos, salgadinhos, biscoitos e outros produtos à base de grãos altamente refinados. Essa é uma das causas principais que levaram muitos países, em particular aqueles em rápido desenvolvimento, à transição nutricional: passando de uma alta prevalência de subnutrição para um aumento de sobrepeso e obesidade na população (KENNEDY et al., 2011; JOHNSTON et al., 2014). Tal aumento tem um impacto negativo, não apenas nos sistemas de saúde, mas também implicações econômicas, sociais e ambientais.

Segundo Triches e Schneider (2010), 
[...] com base nessas referências é que o papel do Estado se sobressai como responsável pela construção e consolidação de modelos alimentares diferenciados que possibilitem o enfrentamento de ambas as problemáticas de produção e de consumo.

Quanto aos aspectos nutricionais de alimentos silvestres produzidos nos modelos sustentáveis de produção, embora não comprovado cientificamente, mas cabe ressaltar, ao fato de que, a simples ausência de agrotóxicos na grande maioria destes produtos, já os coloca em vantagem em relação aos produtos alimentares convencionais.

Ademais, a variedade de alimentos produzidos em uma determinada área e os nutrientes que eles contêm, terá impacto direto na saúde humana e no estado nutricional das pessoas que ali vivem (GIZ, 2016).

Desse modo, a alimentação escolar fornece excelente ponto de entrada para alimentos da agrobiodiversidade, pode ser um canal eficaz para introduzir alimentos com alto valor nutricional na alimentação das crianças e, ao mesmo tempo, fornecem um mercado estável para os produtores rurais.

\section{DESENVOLVIMENTO}

A agricultura surge há aproximadamente 10.000 anos atrás, quando a humanidade iniciou um processo de domesticação e transformação de ecossistemas naturais para ecossistemas cultivados. Gradativamente deixando de explorar populações de vegetais e de animais (MAZOYER; ROUDART, 2010). Em um período anterior a agricultura as mulheres eram as responsáveis pela coleta das plantas e com isso, já iniciavam os primeiros sistemas de cultivos com as sementes destas, enquanto que os homens caçavam (SHIVA, 2003).

O sistema de produção tradicional tem evoluído em diferentes contextos e regiões do mundo, apesar disso, podem-se destacar alguns aspectos semelhantes no que tange: a diversidade biológica, ao manejo e conservação dos recursos naturais, 


\section{International Journal of Environmental}

\section{Resilience Research and Science (IJERRS)}

Revista Internacional Resiliência Ambiental Pesquisa e Ciência Sociedade 5.0 Resiliência Ambiental

a agroecossistemas que apresentam resistência contra perturbações e a formas coletivas de organização para acesso dos recursos e distribuição dos benefícios (ALTIERI; TOLEDO, 2011).

Mas mesmo que a agricultura tradicional represente cerca de $60 \%$ das terras cultivadas (ALTIERI, 2012); esse sistema sofre com forte influência dos sistemas agrários modernos, que medram de novas práticas e meios de produção (MAZOYER, ROUDART, 2010).

Desta forma, a agricultura tem sofrido mudanças ao longo do tempo. A passagem da agricultura tradicional para a moderna teve início na chamada Primeira Revolução Agrícola a partir do século XVIII em diversas regiões europeias, com a aproximação entre as práticas agrícolas e pecuárias através de cultivo de plantas forrageiras alternando com o plantio de outras espécies, resultando na elevada produção de alimentos, que possibilitou o abastecimento alimentar e de outras matérias-primas para Revolução Industrial (EHLERS, 2008).

A partir daí, a intensificação e expansão de elementos como: adubos químicos, mecanização, sementes modificadas, resultou na Segunda Revolução Agrícola também denominada de Revolução Verde, que tivera como objetivo aumentar os índices de produtividade, substituindo os modelos de produção tradicional por um conjunto de práticas homogêneas (ELHERS, 2008).

A Revolução Verde contribuiu para o aumento na produção de alimentos. Em contrapartida, há crescentes evidências sobre os impactos negativos dos sistemas modernos de agricultura intensiva sobre o meio ambiente e modo de vida das pessoas, assim como da incapacidade dos mesmos em atender as necessidades nutricionais da crescente população (LEFF, 2006; SARANDÓN; FLORES, 2014).

As áreas cultivadas, o suprimento de alimentos e os hábitos alimentares, de forma geral, se tornaram mais simplificados e homogêneos. A agricultura se tornou o principal propulsor das perdas de paisagens naturais e de biodiversidade (FANZO et al., 2013). Essa substituição da diversidade biológica pela homogeneização dos 


\section{International Journal of Environmental}

\section{Resilience Research and Sciente (IJERRS)}

Revista Internacional Resiliência Ambiental Pesquisa e Ciência Saciedade 5.0 Resiliência Ambiental

sistemas de cultivo resulta em desequilíbrios ecológicos, o que promove a dependência, cada vez maior, das unidades produtivas a insumos externos (SHIVA, 2003; CAPORAL, 2009; ALTIERI, 2012).

Neste contexto de intensa ação humana sobre recursos naturais e degradação do meio ambiente, passam a existir preocupações acerca destes problemas, surge então, a Conferência das Nações Unidas sobre o Meio Ambiente Humano ou então Conferência de Estocolmo em 1972, um marco nas tentativas de melhorar as relações do homem com o Meio Ambiente. É neste momento que vem a tona a noção de ecodesenvolvimento - Crescer sem destruir - (SACHS, 1985) e que mais tarde, em 1987 com o relatório de Brundtland - Nosso futuro comum - evoluiria para a noção de desenvolvimento sustentável (FAVARETO, 2006).

Considerando os problemas expostos na seção anterior, e o novo paradigma do desenvolvimento, torna-se fundamental construir uma argumentação favorável ao desenvolvimento sustentável e a produção de alimentos em escala local ou regional.

A iniciar pela substituição do termo "ecodesenvolvimento" para "desenvolvimento sustentável". Enquanto ecodesenvolvimento trazia a ideia de incompatibilidade entre o crescimento econômico e a proteção ambiental; desenvolvimento sustentável preza pela compatibilidade, defendendo ser possível associar o crescimento econômico com a conservação ambiental (VEIGA, 2005).

O desenvolvimento sustentável tem como linha central a melhoria da qualidade de vida humana dentro dos limites da capacidade de suporte dos ecossistemas. Desta forma contrapõe-se à visão tradicional de desenvolvimento, herdada do século XIX, que privilegie ionizantes em qualquer fase do processo de produção, armazenamento e de consumo, e entre os mesmos, privilegiando a preservação da saúde ambiental e humana.

Ademais, a promoção do desenvolvimento sustentável está diretamente ligada à valorização do meio rural, uma vez que a agricultura familiar apresenta vantagens econômicas, sociais e ambientais, produzindo alimentos carregados de atributos 


\section{International Juurnal of Environmental Resilience Research and Sciente (IJERRS)}

Revista Internacional Resiliência Ambiental Pesquisa e Ciência Saciedade 5.0 Resiliência Ambiental

culturais e locais frente à padronização da produção em grande escala (ICCA, 2012), o que a torna um princípio para o desenvolvimento sustentável.

Com isso, é crucial fortalecer pequenos produtores, mais precisamente cadeias de valor de alimentos formadas por vários pequenos produtores, que tendem a ser mais efetivas na conservação da biodiversidade do que as formadas por poucos grandes produtores.

Com a criação e o fortalecimento de cadeias de valor para esses alimentos, agricultores podem alcançar mercados urbanos e também contribuir para diversificar e melhorar o valor nutricional da alimentação de populações urbanas. Na medida em que a produção de alimentos continua a se concentrar em poucas espécies e variedades de plantas e raças de animais e que as espécies silvestres são cada vez mais ameaçadas, cresce a necessidade de preservar e proteger a identidade desses alimentos para as futuras gerações (DECLERCK et al., 2011).

\section{CONSIDERAÇÕES FINAIS}

Conclui-se que discutir sobre maneiras alternativas de produção de alimentos pela agricultura familiar torna-se crucial, visto que persistem e agravam-se importantes problemas sociais e ambientais relacionados ao sistema agroalimentar atual ameaçando a saúde dos consumidores e a Segurança Alimentar e Nutricional em nosso país.

As desigualdades sociais causadas pela concentração de bens, ligadas à supremacia do capital sobre o trabalho, as crises ambientais em decorrência da intensificação da agricultura em larga escala e os efeitos sobre a qualidade de vida da população têm feito parte dos debates sobre a atuação do Estado no sentido de promover o desenvolvimento sustentável.

A aproximação da agricultura familiar de programas caracterizados pelo consumo regular de alimentos, como o PNAE, parece uma alternativa viável para dar 


\section{International Journal of Envirnnmental}

\section{Resilience Research and Sciente (IJERRS)}

Revista Internacional Resiliência Ambiental Pesquisa e Ciência Saciedade 5.0 Resiliência Ambiental

sustentabilidade à produção e aos produtores, valorizando as práticas agroalimentares locais e os cultivos diversificados. Portanto recomenda-se a realização de estudos que avaliem como se dá a inclusão das PANC no PNAE no sentido da estruturação desse sistema sustentável de produção e distribuição de alimentos, verificando se atende a processos socialmente justos, economicamente viáveis e ambientalmente adequados, possibilitando o crescimento da economia no contexto do desenvolvimento sustentável.

\section{REFERÊNCIAS}

ALTIERI, M. Agroecologia: bases científicas para uma agricultura sustentável. 3.ed.rev.ampl. São Paulo: Expressão Popular; Rio de Janeiro: AS-PTA, 2012.

ALTIERI, M. A.; TOLEDO, V. The agroecological revolution in Latin America: recuing nature, ensuring food sovereignty and empowering peasants. The Journal Peasant Studies, vol.38, n.3, p.587-612, 2011.

ASSIS, R. L. Desenvolvimento Rural Sustentável no Brasil: perspectivas a partir da integração de ações políticas e privadas com base na agroecologia. Economia Aplicada, vol.10, n.1, p.75-89, 2006.

BRASIL. Lei no 11.326, de 24 de julho de 2006. Diário Oficial da União. Brasília, 2006. Disponível em: http://www.planalto.gov.br/ccivil_03/_ato2004-2006/2006/lei/l11326.htm

BRASIL. Lei nำ11.947 de 16 de junho de 2009. Diário Oficial da União. Brasília, 2009. Disponível em: https://www.fnde.gov.br/legislacoes/institucional-leis/item/3345-lei-n\%C2\%BA11947-de-16-de-junho-de-2009

BRASIL. MINISTÉRIO DO DESENVOLVIMENTO SOCIAL E COMBATE À FOME. Marco de referência de educação alimentar e nutricional para as políticas públicas. Brasília, DF: MDS; Secretaria Nacional de Segurança Alimentar e Nutricional, 2012. Disponível em:

http://mds.gov.br/caisan-mds/educacao-alimentar-e-nutricional/marco-de-referencia-de-educacaoalimentar-e-nutricional-para-as-politicas-publicas

BRASIL. MINISTÉRIO DO MEIO AMBIENTE. Cadernos de debate agenda 21 e sustentabilidade. Agenda 21 e biodiversidade, 1992. Disponível em:

http://www.mma.gov.br/estruturas/agenda21/_arquivos/CadernodeDebates9.pdf

CAPORAL, F. R. Agroecologia: uma nova ciência para apoiar a transição a agriculturas mais sustentáveis. In: CAPORAL, F. R.; COSTABEBER, J. A.; PAULUS, G. Agroecologia: uma ciência do campo da complexidade. 1.ed. Brasília: MDA/SAF, v.1. 111 p. - Brasília: 2009.

DECLERCK, F. A J., FANZO, J., PALM, C., \& REMANS, R. Ecological approaches to human nutrition. Food and Nutrition Bulletin, 32(1 Suppl),41-50. 2011. 


\section{International Journal of Environmental}

\section{Resilience Research and Sciente (IJERRS)}

Revista Internacional Resiliência Ambiental Pesquisa e Ciência Saciedade 5.0 Resiliência Ambiental

Deutsche Gesellschaft fur Internationale Zusammenarbeit (GIZ). ValuES. Counting on Nature's

Benefits - Ecosystem Services: What Are They? Factsheet: Food, 2016. Disponivel em: http://www.aboutvalues.net/ecosystem_services/._Acesso em: 25 de Julho 2019.

EHLERS, E. O que é agricultura sustentável. 1ed. São Paulo: Brasiliense, 2008.

FAVARETO, A.S. Paradigmas do Desenvolvimento Rural em questão - do agrário ao territorial. Tese (Doutorado) apresentada ao Programa de Pós- Graduação em Ciência Ambiental da Universidade de São Paulo - USP. São Paulo, 2006.

FANZO, J., HUNTER, D., BORELLI, T., MATTEI, F. Diversifying food and diets. Using agricultural biodiversity to improve nutrition and health. New York, USA: Biodiversity International, 2013. Disponível em:

http://www.bioversityinternational.org/fileadmin/user_upload/online_library/publications/pdfs/Diversifyin g_food_and_diets_1688_01.pdf. Acesso em 25 de julho 2019.

Food and Agriculture Organization of the United Nations. The state of food and agriculture: climate change, agriculture and food security, 2016. Disponivel em: http://www.fao.org/3/a- i6030e.pdf. Acesso em 25 de julho 2019.

GLIESSMAN, S. R. Agroecologia: processos ecológicos em agricultura sustentável. 1ed. Porto Alegre: Editora da UFRGS, 2000.

IPES-Food. From uniformity to diversity: a paradigm shift from industrial agriculture to diversified agroecological systems. International Panel of Experts on Sustainable

Food systems, 2016. Disponível em: http://www.ipes-food.org/images/Reports/ UniformityToDiversityFullReport.pdf. Acesso em 25 de julho 2019.

ICCA - INSTITUTO INTERAMERICANO DE COOPERAÇÃO PARA A AGRICULTURA (Brasil) Economia Verde e Inclusão Socioprodutiva: o papel da agricultura familiar. Documento final do Congresso Virtual Internacional. Reflexões para Rio + 20. Disponível em:

http://www.iica.int/Esp/regiones/sur/brasil/Lists/DocumentosTecnicosAbertos/Attachments/639/MMORelatorioV2-17052012.pdf. Acesso em 25 de julho 2019.

JOHNSTON, J.L., FANZO, J.C., COGILL, B. Understanding Sustainable Diets: A Descriptive Analysis of the Determinants and Processes That Influence Diets and Their Impact on Health, Food Security, and Environmental Sustainability. Adv. Nutr. v.5, p 418-429. 2014.

KENNEDY, G. TERRI, B. MARIE C. Guidelines for measuring household and individual dietary diversity. Food and Agriculture Organization of the United Nations, 2011. Disponível em: http://www.fao.org/fileadmin/user_upload/wa_workshop/docs/FAO-guidelines-dietarydiversity2011.pdf. Acesso em 25 de julho 2019.

KINUPP, V. F.; LORENZI, H. Plantas alimentícias não convencionais (PANC) no Brasil: guia de identificação, aspectos nutricionais e receitas ilustradas. São Paulo: Instituto Plantarum de Estudos da Flora,768p. 2014.

KINNUP, V. F. Plantas Alimentícias Não Convencionais da região metropolitana de Porto Alegre, RS. Tese (Doutorado), Universidade Federal do Rio Grande do Sul, 2007.

LEFF, E. O retorno da ordem simbólica: a civilização da natureza e as estratégias fatais do desenvolvimento sustentado. In: LEFF, E. Racionalidade ambiental: a reapropriação social da natureza. Rio de Janeiro: Civilização Brasileira, 2006.

MAZOYER, M.; ROUDART, L. História das agriculturas no mundo: do neolítico à crise contemporânea. São Paulo: Editora UNESP; Brasília, DF: NEAD, 2010.
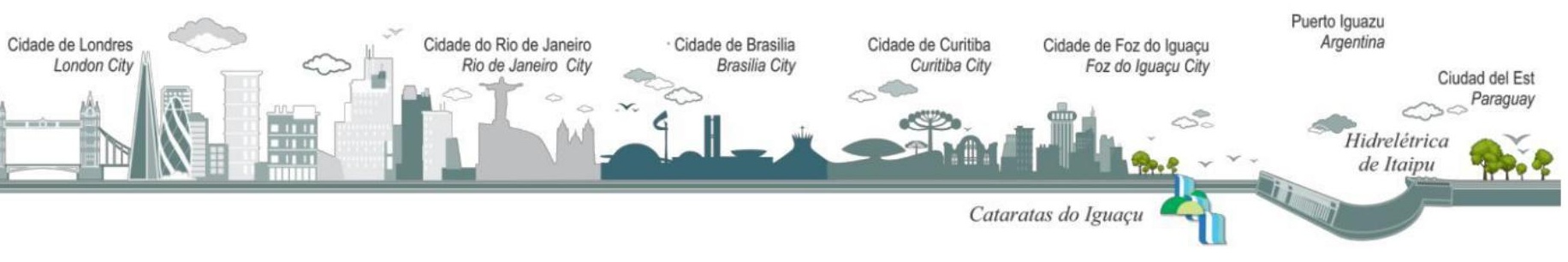


\section{International Juurnal of Environmental}

\section{Resilience Research and Sciente (IJERRS)}

Revista Internacional Resiliência Ambiental Pesquisa e Ciência Sociedade 5.0 Resiliência Ambiental

OLALDE, A. R. Agricultura familiar e desenvolvimento sustentável. Cruzeiro, DF:

CEPLAC. (Radar técnico - artigos técnicos), 2012. Disponível em:

http://www.ceplac.gov.br/radar/Artigos/artigo3.htm. Acesso em 25 de julho 2019.

PADULOSI, S., THOMPSON, J,, RUDEBIER, P. Fighting Poverty, Hunger and Malnutrition with Neglected and Underutilized Species: Needs, Challenges and the Way Forward. Rome, 2013:

Bioversity International. Disponível em: https://www.bioversityinternational.org/

fileadmin/_migrated/uploads/tx_news/Fighting_povert y_hunger_and_malnutrition_with_ neglected_and_underutilized_species_NUS_1671_03.pdf

SEN, A. Desenvolvimento como liberdade. São Paulo: Companhia de Letras, 2010.

SHIVA, V. Monoculturas da mente: perspectivas da biodiversidade e da biotecnologia. São Paulo: Gaia, 2003.

SACHS, I. Estratégias de transição para o século XXI. In: BURSZTYN, M. Para pensar o

Desenvolvimento Sustentável. São Paulo: Brasiliense, p.29-56.1993.

SARANDÓN, S. J.; FLORES, C. C., Agroecologia: o enfoque necessário para uma agricultura sustentável. In: SARANDÓN, S. J.; FLORES, C. C. Agroecología: bases teóricas para o desenho e manejo de agroecossistemas sustentáveis. La Plata: Universidad Nacional de La Plata, p. 41-69. 2014.

SOUZA, F.J.L. Agroecologia como ferramenta para o desenvolvimento sustentável da agricultura familiar. Documento técnico aberto publicado por ICCA - Instituto Interamericano de Cooperação para a Agricultura, 2012. Disponível em:

http://www.iica.int/Esp/regiones/sur/brasil/Lists/DocumentosTecnicosAbertos/Attachments/45 0/Artigo_Agroecologia_como_ferramenta_para_o_desenvolvimento_sustentavel_-_NEAD.pdf. Acesso em 25 de julho 2019.

TRICHES, R. M.; SCHNEIDER, S. Reconstruindo o "elo perdido": a reconexão da produção e do consumo de alimentos através do Programa de Alimentação Escolar no município de Dois Irmãos (RS). Segurança Alimentar e Nutricional, v. 17, n. 1, p. 115, 2010.

VEIGA, J.E. Desenvolvimento sustentável: o desafio do século XXI. Rio de Janeiro: Garamond, 2005. 